\title{
Análisis de materiales didácticos digitales ofertados desde un portal de contenidos abiertos: el caso de Canarias
}

\section{Análise de materiais didáticos disponibilizados em um portal de conteúdos abertos: o caso de Canárias}

\section{Analysis of digital teaching materials provided from an open website: the case of the Canary Islands}

\author{
Carlos José González Ruiz* \\ Elena Chirino Alemán**
}

\begin{abstract}
RESUMEN
En este artículo se ofrece los resultados del análisis de una muestra de los contenidos o materiales didácticos digitales para la Educación Primaria existentes en un portal web denominado Eco Escuela 2.0 impulsado por la administración educativa (Consejería de Educación del Gobierno de Canarias), caracterizado por el acceso y uso libre por el profesorado. El propósito principal es realizar un análisis de materiales dirigido al final de la etapa de educación primaria en diferentes materias. La metodología empleada del estudio consistió en el análisis de contenido. Con esta finalidad se ha elaborado un instrumento-guía para el análisis de la muestra. Los resultados de la investigación detectan actividades que pretenden favorecer la resolución de problemas, la experimentación o la simulación. Su diseño es aceptable aunque mejorable en calidad: algunos elaborados por docentes
\end{abstract}

* Universidad de La Laguna. San Cristóbal de La Laguna, Santa Cruz de Tenerife, España. E-mail: cgonzalezruiz81@gmail.com. https://orcid.org/0000-0003-4269-6221.

** Universidad de Las Palmas de Gran Canaria. Las Palmas de Gran Canaria, Las Palmas, España. E-mail: elena.chirino@ulpgc.es. https://orcid.org/0000-0001-7435-1303. 
por lo que carecen del atractivo visual de otros materiales más comerciales. La mayoría son cerrados y no modificables, creados para unos contenidos y fines concretos aunque sí adaptados a diferentes ritmos de aprendizaje. En general, no ofrecen actividades finales y en varios, estos terminan con la realización de tareas grupales entregables, elaboración de materiales, discusión de ideas o la exposición del trabajo. La diversidad cultural o funcional de género no está presente en estos materiales y aunque el acceso a estos materiales es bueno, se debe mejorar la optimización para todo tipo de pantallas de dispositivos móviles.

Palabras claves: Materiales didácticos digitales. Portal educativo institucional. Análisis de contenido. Recursos educativos. Escuela digital.

\section{RESUMO}

Apresenta os resultados de análise de uma amostra dos conteúdos e materiais didáticos digitais para a Escola Primária, disponibilizados em um portal web denominado Eco Escuela 2.0, desenvolvido pela administração educacional do Governo das Ilhas Canárias, caracterizado pelo acesso e uso livre por professores. O objetivo principal é realizar a análise de materiais destinados à etapa final da educação primária em diferentes disciplinas escolares. A metodologia utilizada foi a análise de conteúdo, utilizando-se um instrumento-guia para examinar a amostra. Os resultados mostraram a presença de atividades que pretendem favorecer a resolução de problemas, a experimentação ou a simulação. Os projetos visuais sugerem a necessidade de melhorias. Eles foram desenvolvidos pelos professores e carecem dos atrativos visuais encontrados em materiais editados comercialmente. A maioria dos materiais não permite modificações, foi criada para conteúdos e objetivos concretos, embora adaptados a diferentes ritmos de aprendizagem. Em geral, não apresentam atividades finais e muitos deles concluem com a realização de atividades em grupo a serem entregues, a elaboração de materiais, a discussão de ideias ou com a exposição do trabalho realizado. A diversidade cultural e de gênero não está presente nos materiais analisados; embora o acesso aos recursos seja bom, deve-se melhorar a sua otimização para todo tipo de tela de dispositivos móveis.

Palavras-chave: Materiais didáticos digitais. Portal educativo institucional. Análise de conteúdo. Recursos educativos. Escola digital.

\footnotetext{
ABSTRACT

This article provides the results of the analysis of a sample of the digital contents or teaching materials for Primary Education existing in a website called Eco Escuela 2.0 promoted by the educational administration
} 
(Educational Counselling of the Canary Islands Government), characterized by the free access and use for the teachers. The main purpose is to perform a material analysis oriented towards the end of the Primary Education in different subjects. The methodology used in the study consisted in content analysis. To this end, an instrument-guide for the analysis of the sample has been prepared. The results of the investigation detect activities that aim to favor the resolution of problems, experimentation or simulation. Its design is acceptable, although qualitatively improvable: some are developed by teachers, thus they lack of the visual appeal of commercial materials. Most are closed and non-modifiable, created for specific contents and purposes, although they are adapted to different learning paces. In general, they do not provide final activities and some of them finish with the accomplishment of deliverable group tasks, elaboration of materials, discussion of ideas or task presentation. The cultural or functional diversity of gender is not present in these materials and although the access is good, optimization must be improved for all types of mobile device screens.

Keywords: Digital teching materials. Institutional educational website. Content analysis. Educational resources. Digital school.

\section{Introducción}

En las últimas décadas del siglo XX y el tiempo transcurrido del siglo XXI, hemos asistido a una transformación social con la irrupción de las tecnologías de la información y la comunicación, que actualmente forman parte de nuestra vida y el ámbito educativo no iba a ser menos. En palabras de Baztán (2014) las administraciones han hecho un gran esfuerzo por ofrecer a la ciudadanía, y a los agentes educativos, familias y alumnado en particular, entornos en los que poder informarse, formarse, compartir e interactuar para la mejora de la calidad educativa y la búsqueda del éxito del proceso de enseñanza-aprendizaje. Además, debemos tomar en consideración que se había asumido que el aprendizaje formal tenía lugar en un aula y con mediación de un profesor aunque según Brazuelo y Gallego (2014) sin tener en cuenta el factor de movilidad. Por tanto, el proceso de aprendizaje fluye a través de diversas localizaciones a través del tiempo que posteriormente se puede transferir a otros contextos completamente distintos.

De este modo, podemos apreciar como las administraciones educativas de las distintas Comunidades Autónomas han ido creando espacios para la difusión de materiales didácticos digitales (en adelante, MDD) en abierto, de forma que los docentes puedan tener los materiales a su alcance, facilitando así su utili- 
zación (BAZTÁN, 2014). Es por ello que el papel de las administraciones en el proceso de integración de las TIC y los MDD en las escuelas es crucial, en cuanto a su responsabilidad de proporcionar la infraestructura y la dotación de los equipos, software, materiales y recursos; y de implementar planes y directrices para la introducción y promoción de su uso en los centros y en promover políticas de asesoramiento y formación del profesorado (véase SANABRIA, ÁLVAREZ y PEIRATS, 2017). Aunque advertimos como ya hicimos en otra ocasión (CHIRINO, ROMERO, CASTRO y ETOPA, 2018), que no basta con tener portales institucionales sino que hay que reflexionar sobre ellos y analizar que nos ofrecen, así como qué aspectos se pueden mejorar.

Ya hace más de una década que Gertrudix (2006) resaltaba la importancia de los portales educativos por sus características como distribuidores de información y almacenes de recursos a la hora de explotar los MDD como recurso en el aula: Pero hay que tener presente tanto las ventajas más relevantes (favorecer el aprendizaje constructivo o dotar de mayor participación y cooperación en tiempo real), como los posibles inconvenientes que se puede encontrar (problemas técnicos, problemas de infraestructura de los centros) o bien la escasa dotación o actualización y mantenimiento de los dispositivos. En España, los recursos tecnológicos tienen una presencia importante en las escuelas. De acuerdo con un estudio de Sánchez-Antolín y Blanco (2016), al menos 85\% de las escuelas tienen acceso Internet en todas las aulas, un $80 \%$ tienen al menos un ordenador para el profesor y más del $50 \%$ de las aulas tienen un ordenador para cada estudiante. Sin embargo, los recursos más novedosos, como son las tabletas, son minoritarios; tan solo el $0,8 \%$ de las aulas dispone de ellos.

Aunque en este sentido, la innovación tecnológica no se traduce directamente en innovación pedagógica, lo que sugiere que en los próximos años las políticas educativas debieran poner más el acento en las transformaciones del modelo de las prácticas educativas y enfatizar menos (aunque sin obviarlo) la dotación de aparatos tecnológicos a las aulas (AREA, 2018). Existen algunos estudios que reflejan esta necesidad de cambio en las prácticas educativas que favorezcan implementar los MDD, puesto que un incremento en las tecnologías disponibles no lleva aparejada la modificación de las prácticas. Es por ello que sigue existiendo con frecuencia el uso de materiales impresos (libros de texto, cuadernillos de trabajo, etc.) y lo que el alumnado realiza con las tecnologías son, principalmente, búsquedas de información o ejercicios on line de modo individual (SÁNCHEZ-ANTOLÍN y BLANCO, 2016). Todo ello a pesar de la cantidad de nuevos MDD que están surgiendo en los últimos años. Sin alcanzar una calidad pedagógica ni tecnológica excepcional, los recursos disponibles resultan de los más recomendables, dado su equilibrio entre lo atractivo de la tecnología y la calidad de los contenidos. Sin embargo, el ecosistema digital educativo digital 
es un espacio en continuo crecimiento donde están disponibles numerosos sitios web, blogs, redes docentes, portales institucionales, de empresas editoriales y otros agentes e instituciones que ofrecen una muy abundante de cantidad objetos, productos, servicios, recursos y herramientas online destinadas a su utilización didáctica pero acompañada de la problemática por la falta de actualización de los portales institucionales (AREA, 2017; SANTANA, EIRÍN y MARÍN, 2017) existiendo en consecuencia, una amalgama a modo de cajón de sastre de productos educativos digitales que complica su identificación y definición.

Los nuevos materiales didácticos emergen como una escenografía abierta y virtual donde docentes y estudiantes tienen el potencial de articular proyectos y experiencias escolares basados en pedagogías para el aprendizaje activo (AREA, 2017). Por tanto, estos nuevos recursos traen consigo nuevas funciones y usos diferentes suponiendo distintos modos de concebir y entender la enseñanza en unos y otros. En palabras de Cepeda, Gallardo y Rodríguez (2017), en algunos casos, se trata de materiales que han sufrido escasas modificaciones o transformaciones respecto a los materiales impresos en cambio otros materiales presentan una gran complejidad tanto desde el punto de vista del diseño como en sus implicaciones pedagógicas. A esto va unido que estos nuevos materiales o recursos educativos online adoptan distintas tipologías tanto en su origen como en su formato, donde se pueden identificar diferentes tipos de recursos educativos, entre los cuales destacan: (a) libros digitales educativos, (b) materiales educativos gamificados y (c) materiales digitales autoconstruidos.

Los primeros responden a una visión estructurada del conocimiento, similar a los libros de texto en papel, pero incorporando la interactividad, el lenguaje audiovisual e iconográfico y la hipertextualidad. La funcionalidad principal de dichos materiales es la presentación de la información a los estudiantes de forma atractiva y facilitadora de su recepción (AREA y MARZAL, 2016). Los segundos (también conocidos como serious games) ofrecen experiencias abiertas y flexibles de aprendizaje apoyadas en las aportaciones de los videojuegos (AREA, GONZÁLEZ y MORA, 2015) mientras que los últimos, elaborados por docentes a partir de la yuxtaposición y mezcla de objetos digitales (un video, un blog, un portal web, una presentación multimedia, una actividad online, o cualquier otro producto) para que su alumnado desarrolle tareas de aprendizaje en torno a los mismos.

En general, se trata de materiales que son desconocidos para el profesorado y demandan una importante participación para el alumnado y las familias en la toma de decisiones sobre los mismos. Igualmente, los resultados de investigación recientes de distintos autores (RODRIGUEZ, BRUILLARD y HORSLEY, 2015; GOMEZ, BRAGA y RODRIGUEZ, 2016) ponen de relieve importantes carencias tanto en su contenido como en su diseño formal y una buena parte de 
estos recursos no han sido ni evaluados ni experimentados, lo que determina un importante grado de incertidumbre en relación a sus posibilidades de utilización didáctica. Esto a pesar de que la inmensa mayoría del alumnado de educación primaria y secundaria son una generación familiarizada con la tecnología digital, motivada y que demanda expresamente el uso de los recursos tecnológicos en la escuela, pero al mismo tiempo son críticos con el nivel de alfabetización digital alcanzado por sus profesores (AREA, CEPEDA y FELICIANO, 2018).

Partiendo de este contexto, nos proponemos presentar el análisis de algunos de los MDD dirigido principalmente al final de la etapa de educación primaria en sus diferentes materias desde el Portal Eco Escuela 2.0 de la Comunidad Autónoma de Canarias, que por un lado ofrece el bloque de recursos educativos digitales atendiendo a materiales desestructurados con los que los docentes puede elaborar los suyos adaptándolos a las necesidades de su práctica y así favorecer el perfil docente de creadores de contenidos; $y$ en el bloque de servicios en la red, desde donde se ofertan una serie de recursos con los cuales los docentes puede elaborar blog, vídeos, radio, etc. La justificación de la necesidad de realizar una evaluación de los MDD, entre otras razones están las señaladas por CEPEDA, GALLARDO y RODRÍGUEZ (2017), puesto que debe servirnos para reflexionar y pensar cuál es el papel de estos recursos en el currículo educativo y cuáles son los principales obstáculos que existen para su implementación en los centros educativos.

\section{Objetivos y pregunta de investigación}

\section{Pregunta de investigación}

La pregunta de investigación a responder en dicho artículo es la siguiente:

- ¿Cuál o cuáles son las características tecnológicas, pedagógicas y de evaluación/seguimiento que poseen los MDD para la Educación Primaria en el portal educativo Eco-Escuela 2.0 del Gobierno de Canarias?

Además, y de forma más específica se intenta dar respuesta a las siguientes preguntas:

- ¿Cuál o cuáles son las características tecnológicas que poseen los MDD para la Educación Primaria?

- ¿Cuál o cuáles son los modelos didácticos que subyacen en los materiales educativos para la Primaria?

- ¿Cuál o cuáles son los modelos socio comunicativos que favorecen los MDD entre los docentes y estudiantes? 
El objetivo principal de dicho artículo se corresponde con la pregunta de investigación expuesta con anterioridad y que se corresponde con el proyecto de investigación titulado "La escuela de la sociedad digital: análisis y propuesta para la producción y uso de los contenidos digitales educativos-EDU2015-65493-R". Esta referencia no solo es vital para contextualizar este trabajo sino para comprender la estructura y decisiones tomadas en cuanto a la formulación de objetivos y metodología. Con dicho estudio se busca comprender el proceso de creación y uso de diferentes materiales didácticos digitales que se ofrecen desde dicho portal. Procedemos a explicitar el mismo:

\section{Objetivo principal}

- Análisis de una muestra de materiales didácticos digitales ofertados desde el portal Eco-Escuela 2.0 impulsado por la administración educativa (Consejería de Educación del Gobierno de Canarias).

\section{Metodología}

El proceso metodológico llevado a cabo en dicho artículo se corresponde con el realizado en dos artículos de las mismas características y que comparten las preguntas de investigación y los objetivos a responder con dicho estudio (CEPEDA, GALLARDO y RODRÍGUEZ, 2017; MATO, CASTRO y PEREIRO, 2018). Entendemos al análisis de contenido como la metodología cualitativa apropiada para analizar las características de los materiales digitales partiendo de la idea transmitida por Bardin (2002: 29) de que "el análisis de contenido es un conjunto de técnicas de análisis comunicativas usando procedimientos sistemáticos y objetivos para describir los contenidos de los mensajes". Para ello, se han seguido diferentes pasos que procedemos a mostrar a continuación.

\section{Muestra}

Para el análisis de materiales o recursos online dentro de las plataformas nos hemos centrado únicamente en aquellos productos digitales elaborados intencionalmente para guiar o apoyar un proceso de enseñanza-aprendizaje completo 
utilizando los recursos online. Veamos a continuación de un modo detallado cuáles han sido los criterios adoptados para la selección de la muestra analizada.

\section{Criterios detallados de la muestra escogida}

\section{Materiales didácticos digitales}

Con respecto a los materiales didácticos digitales (MDD), se han analizado un total de 4, pertenecientes a la plataforma Eco-escuela 2.0 (Consejería de Educación y Universidades del Gobierno de Canarias). Entendemos, al igual que Cepeda, Gallardo y Rodríguez (2017) que es necesario clarificar previamente los tipos de materiales digitales para poder determinar qué materiales se seleccionan (AREA, 2017):

- Objeto digital. Es un archivo digital que porta contenido, información y/o conocimiento. Cuando están almacenados de forma organizada constituyen un repositorio de objetos digitales.

- Objeto digital de aprendizaje. Es un tipo particular de objetos digitales creados con intencionalidad didáctica a corto plazo que implican alguna acción del estudiante. Adoptan, en la mayor parte de las ocasiones, el formato de actividades o ejercicios aislados.

- Material didáctico digital (MDD). Es un paquete estructurado didácticamente de objetos digitales en línea dirigido a facilitar al alumnado el desarrollo de experiencias de aprendizaje en torno a una unidad de saber o competencia. Son materiales para la educación formal ya que en los mismos subyace una propuesta o proyecto de desarrollo curricular. Este tipo de materiales educativos tienen una amplia y larga tradición en nuestros estudios y ya conocemos muchas de sus dimensiones de análisis producidos en soporte de papel (libros de texto) o multimedia (CD-ROM, DVD y similares).

- Materiales profesionales de docentes. Son el conjunto de objetos digitales que ofrecen programaciones, experiencias prácticas, propuestas elaboradas de intervenciones educativas, espacios de publicación del profesorado (blogs, wikis,). Son recursos de interés para el profesorado en su autoformación y mejora profesional.

- Apps, herramientas y plataformas online. Es software. A veces son de propósito general y en otras ocasiones específicamente creados para el ámbito educativo. Hay cientos y constantemente están en evolución y crecimiento (p. 4-5). 


\section{Criterios de selección de la muestra con respecto a los materiales didácticos digitales}

Los criterios de selección de los recursos digitales fueron los siguientes:

- Destinados a $5^{\circ}$ o $6^{\circ}$ de Educación primaria.

Se seleccionan los MDD que supongan propuestas estructuradas para uso del alumnado para su aprendizaje y no guías o material de apoyo dirigidas al profesorado.

- Organizado en torno a las asignaturas Lengua y Literatura Castellana, Matemáticas, Ciencias de la Naturaleza y Ciencias Sociales, y algún material de otras asignaturas.

- Que se puedan analizar todas las dimensiones contempladas en el instrumento de análisis que se describe en este trabajo: características identificadoras del material o recursos educativos, estructura del material, dimensión tecnológica, dimensión de diseño, dimensión pedagógica, dimensión de contenido, evaluación y seguimiento.

\section{Instrumentos y procesos de análisis}

Las técnicas e instrumentos usados en dicho estudio fueron los siguientes. Se describe el proceso de elaboración, aplicación y análisis de los mismos:

- Entrevista semiestructurada al responsable del portal Eco-Escuela 2.0.

- Elaboración de un instrumento (guion) ad-hoc usando el siguiente procedimiento:

- A partir de un borrador de instrumento basado en trabajos de investigación previos sobre análisis de materiales didácticos se fue trabajando en un segundo borrador.

- El segundo borrador fue sometido al juicio de 8 personas expertas en materiales didácticos, en concreto cinco expertas internacionales (de Brasil, Portugal, y Argentina) y tres expertos nacionales (de Barcelona, Valencia y Murcia).

- Se elaboró el instrumento definitivo incorporando sugerencias del panel de personas expertas consultadas. En el mismo, se recogen diferentes dimensiones que procedemos a mostrar a continuación: material o recursos educativo, estructura del material, dimensión tecnológica, 
dimensión de diseño, dimensión pedagógica, dimensión de contenido, evaluación y seguimiento, aspectos positivos/fortalezas y aspectos negativos/debilidades.

- Análisis de los materiales didácticos seleccionados. Siguiendo las sugerencias relacionadas con el análisis de contenido, se ha procedido al análisis de los materiales indicados anteriormente.

- Elaboración de los informes correspondientes y puesta en común de los equipos de investigación. Los informes se elaboran siguiendo la misma estructura que la de los protocolos de evaluación y se ponen en común siguiendo el procedimiento ya comentado anteriormente.

\section{Resultados}

A continuación procederemos a describir el análisis realizado sobre los materiales didácticos digitales. Nos hemos centrado en las características más destacables de los mismos, poniendo especial hincapié en las siguientes dimensiones, las cuáles nos ayudarán a responder a la pregunta de investigación planteada en dicho estudio:

- Características tecnológicas/diseño del material.

- Dimensión pedagógica.

- Evaluación y seguimiento.

Antes de empezar a realizar una descripción más exhaustiva de los diferentes materiales, se ofrecen (ver Tabla 1, a continuación) los datos identificativos de los materiales didácticos digitales analizados: 


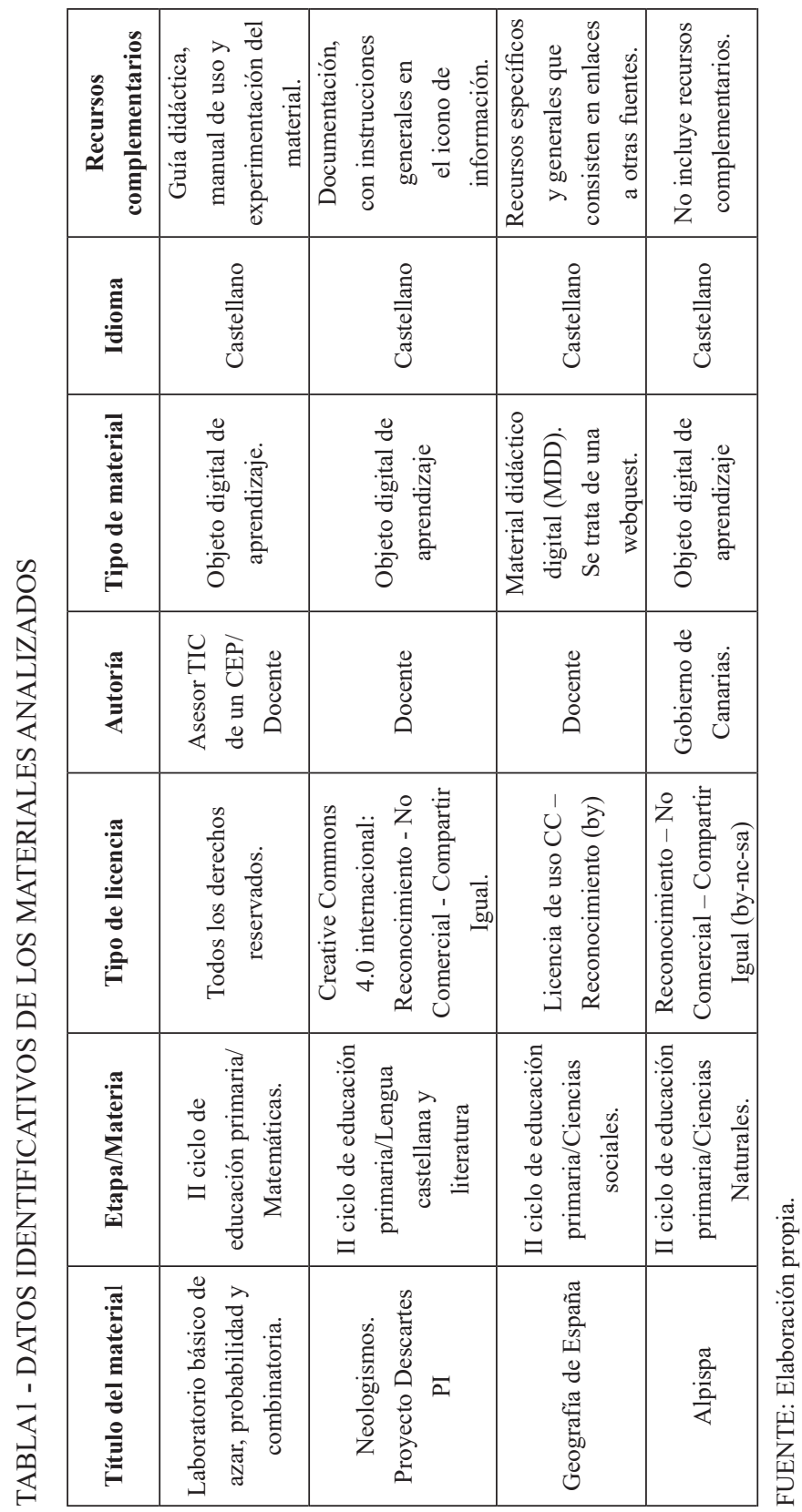


Nos encontramos ante cuatro materiales didácticos digitales destinados a la misma etapa, en este caso, el II ciclo de educación de primaria, que se corresponde con los cursos de $5^{\circ}$ y $6^{\circ}$ de primaria del curriculum educativo español. Pasemos a analizar más detalladamente algunos de sus datos:

- Materia: Nos encontramos con materiales destinados a diferentes asignaturas/materias, en este caso: Matemáticas, Lengua Castellana y Literatura, Ciencias sociales y Ciencias naturales.

- Tipo de licencia: Tres de los cuatro materiales analizados poseen una licencia creative commons, es decir, que el usuario puede hacer uso del material educativo así como también lo puede compartir, siempre y cuando no haga un uso comercial del mismo. Por otra parte, nos encontramos, el material destinado al aprendizaje de las matemáticas tiene "Todos los derechos reservados".

- Autoría: La mayoría de los materiales analizados en dicho estudio han sido creados por el profesorado, a excepción del material destinado a las ciencias naturales, que ha sido elaborado por el Gobierno de Canarias.

- Tipo de material: Los tipos de materiales analizados se corresponden con los expuestos anteriormente en el apartado metodológico. En esta ocasión nos encontramos con tres materiales que se incluyen dentro de la categoría: objeto digital de aprendizaje. Dichos materiales están creados con intencionalidad didáctica a corto plazo que implican alguna acción del estudiante. Adoptan, en la mayor parte de las ocasiones, el formato de actividades o ejercicios aislados. Por otra parte, también se ha analizado un material que adopta la categoría de Material didáctico digital (MDD), que podríamos definir como paquete estructurado didácticamente de objetos digitales en línea dirigido a facilitar al alumnado el desarrollo de experiencias de aprendizaje en torno a una unidad de saber o competencia. Son materiales para la educación formal ya que en los mismos subyace una propuesta o proyecto de desarrollo curricular.

- Idioma: Todos comparten el mismo idioma: el castellano. No ofrece la posibilidad de utilizarlo en otra lengua.

- Recursos complementarios: Tres de los cuatro recursos analizados ofrecen recursos complementarios, ofreciendo información extra sobre la utilización del recurso en cuestión. 


\section{Características tecnológicas/diseño de los MDD}

En lo que concierne a las características tecnológicas y de diseño, los materiales analizados presentan una serie de rasgos que presentamos a continuación. Nos encontramos con cuatro materiales con una navegabilidad y usabilidad muy sencilla por parte del usuario. Con respecto al uso del material en otras plataformas (Pizarras digitales Interactivas, dispositivos móviles, etc.), tres de los cuatro materiales pueden ser usados en dichos dispositivos, salvo el destinado a ciencias sociales, que sólo se puede usar a través de un ordenador y un navegador WEB. En cuanto al nivel de interactividad que ofrecen los materiales, encontramos bastantes diferencias entre ellas, ya que los destinados a Matemáticas y Ciencias Naturales tiene una interactividad alta, mientras que los otros dos materiales la poseen baja y moderada. Todos los materiales ofrecen un diseño que facilita la comprensión de los contenidos por parte del alumnado, así como un diseño adecuado a las características psicoevolutivas del alumnado destinatario.

\section{Modelos didácticos/pedagógicos que subyacen al material.}

¿Cuál es el modelo pedagógico que subyace al material? En este apartado, a través de la Tabla 2, se describe las diferentes características de cada uno de los materiales analizados.

\section{Evaluación y seguimiento}

Ningún material analizado detalla criterios y estrategias de evaluación. Sin embargo, en los materiales destinados a las materias de Matemáticas y Lenguaje, si ofrecen distintas actividades de evaluación, siendo común las actividades autoevaluación en la que el alumnado debe responder una serie de preguntas (tipo cuestionario), después de haber estudiado los contenidos que se le presentan. El material correspondiente a la asignatura de matemáticas también ofrece la posibilidad de que el alumnado resuelva una serie de problemas, en donde se incluye una situación problemática y el estudiante responde a un cuestionamiento puntual. 


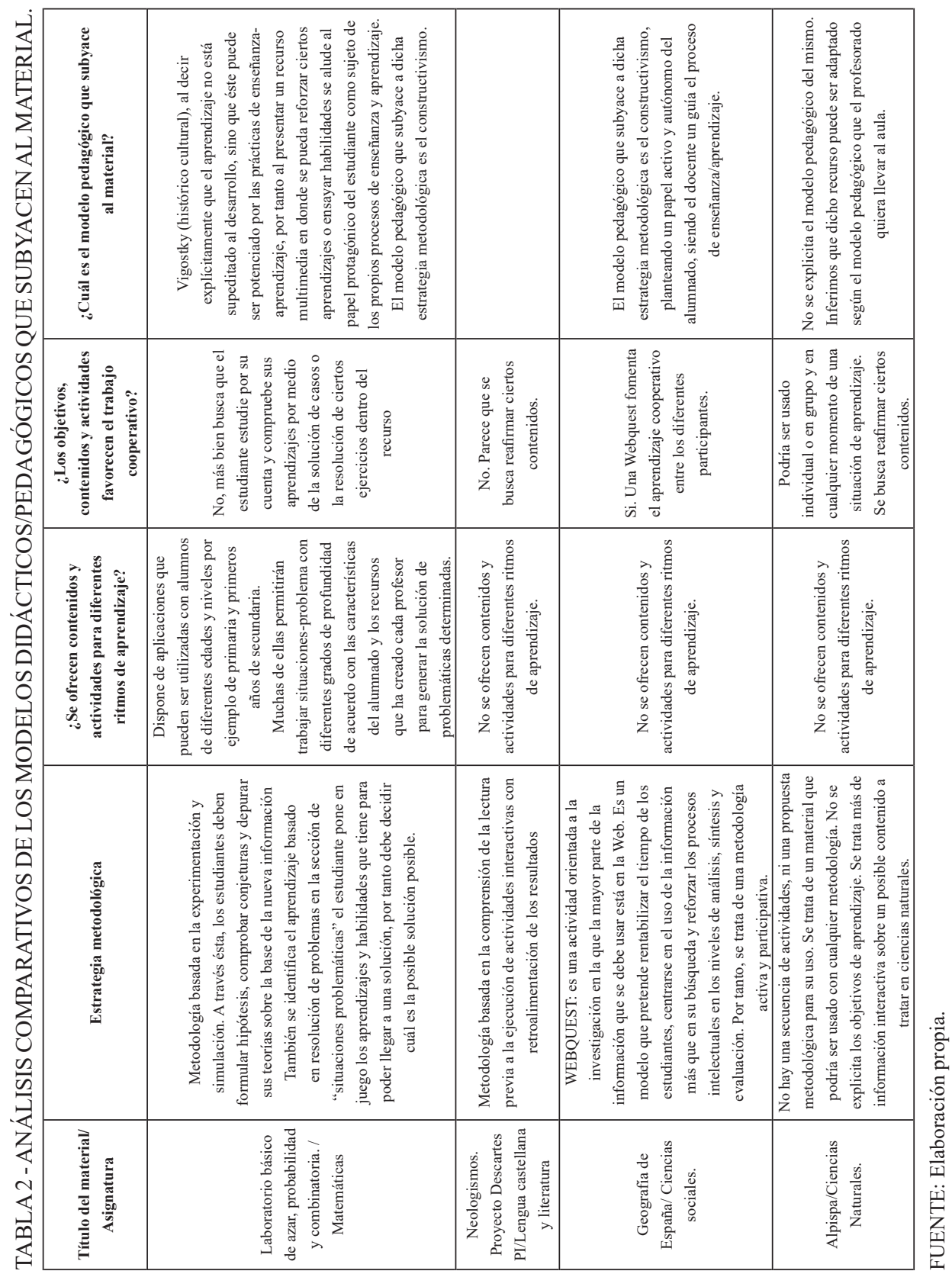


Tras realizar dicha descripción del análisis de los materiales didácticos digitales insertados en el portal Eco-Escuela 2.0 del Gobierno de Canarias (España), se mostrarán las conclusiones que hemos inferido a la luz de los resultados encontrados.

\section{Conclusiones}

El interés de los medios y su evaluación está en la correlación directa que tiene con otros elementos del curriculum educativo (GÁRCIA-VALCÁRCEL y TEJEDOR, 2009). Durante los últimos años, los materiales didácticos están sufriendo una metamorfosis (AREA, 2017), que están haciendo replantear a multitud de agentes educativos el papel que juegan en los mismo así como los procesos llevados a cabo.

Cada día hay mayor oferta educativa en cuanto a materiales y medios para la enseñanza. Igualmente ocurre con la incorporación de herramientas provenientes de la WEB 2.0, los recursos en abiertos y otras iniciativas desarrolladas en los últimos años. Actualmente se realizan evaluaciones sobre diferentes medios didácticos, a destacar:

- Evaluación de material impreso y libros de texto.

- Evaluación de los materiales audiovisuales.

- Evaluación de materiales didácticos digitales.

El principal reto de los agentes educativos, en especial el profesorado, no será la falta de medios porque hay y habrá una cantidad de ingente de recursos. Los materiales didácticos analizados hacen repensar la labor del docente. Viendo las características de estos, entendemos que el papel del profesorado debe consistir en seleccionar aquellas piezas, trozos u objetos digitales que considere pertinentes o con potencial educativo para presentárselos a su alumnado. Por otro lado, podemos observar como la autoría de los materiales didácticos digitales analizados en dicho trabajo se corresponde con el profesorado. Esto concuerda con varios estudios realizados en los últimos años (SANABRIA, ÁLVAREZ y PEIRATS, 2017; GÓNZÁLEZ, MARTÍN y VEGA, 2018). El papel del docente, como creador de materiales didácticos, es una de las características más interesantes encontradas en las últimas investigaciones. La posibilidad que nos dan las TIC de convertirnos en autores es una cuestión para analizar en los próximos años.

Entre las características generales que mostraron los MDD de los portales institucionales fueron las siguientes: 
- A pesar de que existe un mejor diseño de los portales institucionales y aparece una gran diversidad de MDD, se hace necesaria una actualización constante de los mismos para poder ir en consonancia con los avances de las TIC y aprovecharlos de una manera más significativa en los entornos educativos.

- La velocidad de carga para acceder a estos materiales es buena en todos los recursos analizados. La tecnología escogida para todos estos materiales es el HTML o HTML5, permitiendo acceder al recurso elaborado desde cualquier navegador web. Esto hace que sean accesibles desde distintas plataformas (Windows, Mac OS, Android, iOS, etc.). Aunque esto no quiere decir que estén optimizados para todo tipo de pantallas. Esto es algo que habría que mejorar, teniendo en cuenta que el gran uso que se hace en la actualidad de dispositivos móviles.

- El análisis de los materiales escogidos de la plataforma Escuela 2.0 muestra que su diseño, en la mayoría de los casos, es aceptable, aunque podría ser de mayor calidad. Varios de los materiales escogidos tienen un diseño artesanal (elaborado por propios docentes) por lo que no son tan atractivos visualmente como otros materiales comerciales desarrollados con otros medios.

- La mayoría de los materiales pretenden promover o favorecer la resolución de problemas, la búsqueda de información, la formulación de hipótesis, la experimentación o la simulación. Otros materiales, en cambio, no se identifican con ninguna metodología en concreto pues se basan en la realización de ejercicios o en la comprensión de textos.

- Por lo general, los materiales de ambos portales no ofrecen actividades y contenidos adaptados a diferentes ritmos de aprendizaje, dejándose esta tarea al propio docente al incorporar a sus clases los materiales. Tampoco es común encontrar materiales que sean modificables. Son recursos cerrados creados para unos contenidos y fines concretos.

- La diversidad cultural no está presente en este tipo de materiales. Lo mismo ocurre con la diversidad funcional de género, sexual o etaria, pues no es habitual el uso de contenido icónico que haga referencia a estas diferencias. Solo se observa en algunos casos.

- En muchas ocasiones no se cuenta con actividades finales, pues todo el material se basa en ejercicios o en desarrollar distintas actividades. En varios materiales, estos terminan con la realización de tareas grupales entregables, elaboración de materiales, discusión de ideas o la exposición del trabajo elaborado frente al resto del alumnado. 
Para concluir, nos gustaría realizar una serie de comentarios en relación con las líneas de investigación futuras. Los materiales didácticos digitales, como hemos visto, tanto a través de los resultados ofrecidos, como de las referencias consultadas, están cambiando de una manera muy radical, en todos los aspectos: contenidos, facilitación de la comprensión y el aprendizaje, nivel de interactividad y adaptabilidad del estudiante, aspectos técnicos y estéticos, coste económico/distribución, autorías, etc. Esto nos hace repensar la manera en la que hasta ahora hemos venido evaluando los diferentes materiales didácticos durante las últimas décadas. La cantidad ingente de recursos que existen a nuestra disposición en las diferentes plataformas, tanto públicas, como privadas, exige de una actualización, tanto de los investigadores, como de los docentes que lo llevan a la práctica de aula. La velocidad de las tecnologías de la información y la comunicación nos está llevando a crear multitud de materiales sin una reflexión previa que lo acompañe, de ahí que la calidad de los mismo y su contenido no sea muy buena. Por todo ello se requiere de una formación inicial y permanente, que permita, a todos los agentes educativos, realizar materiales didácticos digitales que ayuden a mejorar los procesos de enseñanza/aprendizaje.

\section{REFERENCIAS}

AREA, M. (2017). La metamorfosis digital del material didáctico tras el paréntesis Gutenberg. Revista Latinoamericana de Tecnología Educativa, 16(2).

AREA, M., y MARZAL, M. A. (2016). Entre libros y pantallas. Las bibliotecas escolares ante el desafío digital. Profesorado, 20(1): 227-242

AREA, M., CEPEDA, O. y FELICIANO, L. (2018). El uso escolar de las TIC desde la visión del alumnado de Educación Primaria, ESO y Bachillerato. Educatio XXI, 36(2), 229-276.

AREA, M., GONZÁLEZ, C. y MORA, C. (2015). Beyond Textbooks. Educational Digitals Texts and Gamification of Learning Materials. En: Rodriguez, J., Bruillard, E. y Horsley, M. (Eds.): Digital Textbooks, What's New? Servizo de Publicacións da USC/ IARTEM. Santiago de Compostela, 265-268.

BARDIN, L. (2002). El análisis de contenido. Editorial Akal.

BAZTÁN, M. (2014). Los materiales didácticos digitales en la enseñanza no universitaria española. Análisis comparativo. Valencia: Ediciones Universitat Politécnica de València. 
BRAZUELO, F. y GALLEGO, D. (2014). Estado del Mobile Learning en España. Educar em Revista, 4, 99-128.

CEPEDA ROMERO, O., GALLARDO, I. M. y RODRÍGUEZ RODRÍGUEZ, J. (2017). La evaluación de los materiales didácticos digitales. Revista Latinoamericana de Tecnología Educativa, 16(2).

CHIRINO, E., ROMERO, K. E., CASTRO, J. J. y ETOPA, M. P. Análisis de portales con materiales didácticos digitales: Procomún y EcoEscuela 2.0. En: LÓPEZ, E., COBOS, D., MARTÍN, A. H., MOLINA, L. y JAÉN, A. (2018). Experiencias pedagógicas e innovación educativa. Aportaciones desde la praxis docente e investigadora. pp.3161-3170.

GARCÍA-VALCÁRCEL, A., y TEJEDOR, F. J. (2009). Evaluación de medios didácticos y proyectos TIC. En: DE PABLOS, J. (Coord.). Tecnología Educativa, la formación del profesorado en la era de Internet. (pp. 271-301). Málaga: Aljibe.

GERTRUDIX, F. (2006). Los portales educativos como fuente de recursos materiales. Icono 14. Revista de Comunicación y Nuevas Tecnologías, 7.

GOMEZ, M. A., BRAGA, T. y RODRIGUEZ, J. (2016). Balance y análisis sobre la investigación del texto escolar y los medios digitales. Memorias de la Conferencia Regional para América Latina de la IARTEM. Colombia: Universidad Tecnológica de Pereira.

MATO, D., CASTRO, M. M. y PEREIRO, M. C. (2018). Análisis de materiales didácticos digitales para guiar y/o apoyar el proceso de enseñanza-aprendizaje de las matemáticas. (a)tic Revista D'Innovació Educativa, 20, 72-79.

RODRIGUEZ, J., BRUILLARD, E. y HORSLEY, M. (Eds). (2015). Digital Textbooks, What's New? Santiago de Compostela: IARTEM/Servizo de Publicacions USC.

SANABRIA, A. L., ÁLVAREZ, Q. y PEIRATS, J. (2017). Las políticas educativas en la producción y distribución de materiales didácticos digitales. Revista Latinoamericana de Tecnología Educativa, 16(2), 63-77.

SÁNCHEZ-ANTOLÍN, P. y BLANCO, M. (2016). La política TIC de la Comunidad de Madrid: la perspectiva del profesorado. Revista Latinoamericana de Tecnología Educativa, 15(1), 45-58. https://doi.org/10.17398/1695-288X.15.1.45

SANTANA, P. J., EIRÍN, R. y MARÍN, D. (2017). Análisis y valoración de portales institucionales en España. Los casos de Canarias, Galicia y Valencia. Revista Latinoamericana de Tecnología Educativa, 16(2), 29-48.

Texto recibido el 22/07/2019.

Texto aprobado el 30/08/2019. 